Electronic Supporting Information (ESI)

\title{
Acid-induced, oxygen-atom defect formation in reduced polyoxovanadate-alkoxide clusters
}

Eric Schreiber ${ }^{1}$, Brittney E. Petel ${ }^{1}$, Ellen M. Matson*1

1Department of Chemistry, University of Rochester, Rochester, New York 14627, United States

Table of Contents:

Experimental Methods.

Figure S1. ${ }^{1} \mathrm{H}$ NMR Spectrum of reaction between $1-\mathbf{V}_{6} \mathbf{O}^{2-}$ and $\left[\mathrm{HNEt}_{3}\right] \mathrm{BF}_{4}$

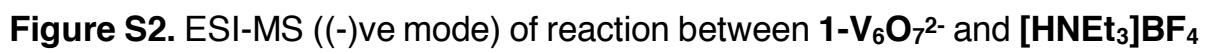

Figure S3. ESI-MS ((-)ve mode) of $3-\mathbf{V}_{6} \mathbf{O}_{6}{ }^{1-}$

Figure S4. IR Spectra of $3-\mathrm{V}_{6} \mathrm{O}_{6}{ }^{1-}, \mathrm{V}_{6} \mathrm{O}_{6}\left(\mathrm{OCH}_{3}\right)_{12^{1-}}$, and $\mathrm{V}_{6} \mathrm{O}_{6}\left(\mathrm{OC}_{2} \mathrm{H}_{5}\right)_{12^{0}}$ S4

Figure S5. Electronic Absorption Spectrum of $3-\mathrm{V}_{6} \mathrm{O}_{6}{ }^{1-}, \mathrm{V}_{6} \mathrm{O}_{6}\left(\mathrm{OCH}_{3}\right)_{12^{1-}}$, and $\mathrm{V}_{6} \mathrm{O}_{6}\left(\mathrm{OC}_{2} \mathrm{H}_{5}\right)_{12^{0}}$. S5

Table S1. IR and Electronic Absorption parameters S5

Figure S6. Cyclic Voltammogram of $\mathbf{1}-\mathbf{V}_{6} \mathbf{O}_{7^{2-}}$ S6

Table S2. Redox Potentials of $1-\mathrm{V}_{6} \mathrm{O}_{7}{ }^{2-}$ S7

References S7 


\section{Experimental Methods}

General Considerations. Unless otherwise stated, all manipulations were carried out in the absence of water and oxygen in a UniLab MBraun inert atmosphere glovebox under a dinitrogen atmosphere. Glassware was oven dried for a minimum of 4 hours and cooled in an evacuated antechamber prior to use in the glove box. Anhydrous methanol and ethanol were purchased from Sigma-Aldrich and stored over activated $4 \AA$ molecular sieves purchased from Fisher Scientific. All other solvents were deoxygenated and dried on a Pure Process Technology Glass Contour System and stored over activated $4 \AA$ molecular sieves purchased from Fisher Scientific. [ ${ }^{\mathrm{n} B u_{4} N} \mathrm{~N}_{\mathrm{PF}_{6}}$ was purchased from Sigma-Aldrich, recrystallized three times from hot ethanol, and stored under dynamic vacuum for at least one day prior to use. Tetrafluoroboric acidether complex was purchased from Sigma Aldrich and used as received. Triethylamine was purchased from Fisher Scientific, dried over $\mathrm{KOH}$, and distilled prior to use. $\left[{ }^{\mathrm{nBu}} \mathrm{N}_{4}\right]_{2}\left[\mathrm{~V}_{6} \mathrm{O}_{7}\left(\mathrm{OC}_{2} \mathrm{H}_{5}\right)_{12}\right]\left(\mathbf{1}-\mathrm{V}_{6} \mathbf{O}_{7^{2-}}\right)^{1},\left[{ }^{n} \mathrm{Bu}_{4} \mathrm{~N}\right]\left[\mathrm{V}_{6} \mathrm{O}_{7}\left(\mathrm{OC}_{2} \mathrm{H}_{5}\right)_{12}\right]\left(\mathbf{2}-\mathbf{V}_{6} \mathbf{O}_{\mathbf{7}^{1-}}\right)^{1}$, and $\left[\mathrm{V}_{6} \mathrm{O}_{6}\left(\mathrm{OC}_{2} \mathrm{H}_{5}\right)_{12}\right]^{0}{ }^{2}$ were prepared as previously reported. ${ }^{1} \mathrm{H}$ NMR spectra were recorded at $400 \mathrm{MHz}$ on a Bruker DPX-400 spectrometer at 9.4 Tesla while locked on to deuterated solvent. $\mathrm{CD}_{3} \mathrm{CN}$ was purchased from Cambridge Isotope Laboratories, degassed by three freeze-pump-thaw cycles, and stored over $4 \AA ̊$ molecular sieves. Mass spectrometry analyses were performed on an Advion Expression ${ }^{\mathrm{L}}$ Compact Mass Spectrometer equipped with an electrospray probe and an ion-trap mass analyser (instrument error: $\pm 0.1 \mathrm{amu}$ ). Direct injection analysis was employed in all cases with a sample solution in acetonitrile. Infrared (FTIR, ATR) spectra of complexes were recorded on a Shimadzu IRAffinity-1 Fourier Transform Infrared Spectrophotometer and are reported in wavenumbers (cm1). Electronic absorption measurements were recorded at room temperature in anhydrous dichloromethane in a sealed $1 \mathrm{~cm}$ quartz cuvette with an Agilent Cary 60 UV-Vis spectrophotometer. Elemental analyses were performed on a PerkinElmer 2400 Series II Analyzer at the CENTC Elemental Analysis Facility, University of Rochester.

Synthesis of $\left[\mathrm{HNEt}_{3}\right] \mathrm{BF}_{4}$. The preparation of $\left[\mathrm{HNEt}_{3}\right] \mathrm{BF}_{4}$ was modified from the literature procedure. ${ }^{3}$ Triethylamine was distilled and degassed via three freeze-pump-thaw cycles prior to bringing into a $\mathrm{N}_{2}$-filled glovebox. A $20 \mathrm{~mL}$ scintillation vial was charged with triethylamine (1.4 $\mathrm{mL}, 9.88 \mathrm{mmol}$ ) and diethyl ether $(5 \mathrm{~mL})$. With vigorous stirring, the tetrafluoroboric acid diethyl ether complex $(1.27 \mathrm{~mL}, 9.39 \mathrm{mmol})$ was slowly added dropwise to the triethylamine solution. WARNING: This reaction is highly exothermic and will cause the ether to boil if the acid is added too rapidly! The reaction was stirred for 5 minutes after the immediate precipitation of a white powder. The suspension was filtered and the solid was washed with $3 \times 5 \mathrm{~mL}$ of diethyl ether, quantitatively yielding the product $\left[\mathrm{HNEt}_{3}\right] \mathrm{BF}_{4}$ as a white powder $(95 \%, 1.68 \mathrm{~g}, 8.89 \mathrm{mmol})$. To limit the interaction of $\left[\mathrm{HNEt}_{3}\right] \mathrm{BF}_{4}$ with adventitious water, the solid was stored in the glovebox over $\mathrm{P}_{2} \mathrm{O}_{5}$.

Synthesis of 3-[CoCp$\left.p_{2}\right]\left[V_{6} O_{6}\left(O_{2} C_{5}\right)_{12}\right]\left(3-V_{6} O_{6}{ }^{1-}\right)$. A $20 \mathrm{~mL}$ scintillation vial was charged with $\left[\mathrm{V}_{6} \mathrm{O}_{6}\left(\mathrm{OC}_{2} \mathrm{H}_{5}\right)_{12}\right]^{0}(0.098 \mathrm{~g}, 0.100 \mathrm{mmol})$ and $6 \mathrm{~mL}$ dichloromethane. Cobaltocene $(0.021 \mathrm{~g}, 0.110$ mmol, 1.1 equiv) was added to the solution as a solid. The reaction mixture was stirred for 2 hours at $21^{\circ} \mathrm{C}$, after which the product was filtered and the volatiles were removed under vacuum to yield $3-\mathrm{V}_{6} \mathrm{O}_{6}{ }^{1-}(0.086 \mathrm{~g}, 0.076 \mathrm{mmol}, 76 \%)$. ${ }^{1} \mathrm{H} \mathrm{NMR}\left(400 \mathrm{MHz}, \mathrm{CD}_{3} \mathrm{CN}\right) \delta=29.59,28.54,-1.28$ (fwhh = $176 \mathrm{~Hz}),-2.56(\mathrm{fwhh}=204 \mathrm{~Hz}),-25.59$ (fwhh = $512 \mathrm{~Hz}) \mathrm{ppm}$. FT-IR $\left(\right.$ ATR, $\left.\mathrm{cm}^{-1}\right): 1416$, 1368, 1092, 1049, 953, 891, 858. UV-Vis $\left(\mathrm{CH}_{3} \mathrm{CN}\right): 406 \mathrm{~nm}\left(\varepsilon=1445 \mathrm{M}^{-1} \mathrm{~cm}^{-1}\right), 526 \mathrm{~nm}(\varepsilon=502$ $\left.\mathrm{M}^{-1} \mathrm{~cm}^{-1}\right)$. Elemental analysis for $\mathrm{C}_{36} \mathrm{H}_{73} \mathrm{O}_{18} \mathrm{~V}_{6} \mathrm{NCo} \bullet 1 / 2 \mathrm{C}_{4} \mathrm{H}_{8} \mathrm{O}(\mathrm{MW}=1131.49 \mathrm{~g} / \mathrm{mol})$ Calcd $(\%)$ : C, 37.76; H, 6.42; N, 1.16. Found (\%): C, 38.12; H, 5.90; N 0.86. 
Synthesis of $3-\mathbf{V}_{6} \mathbf{O}_{6}{ }^{1-}$ via acid addition. A $20 \mathrm{~mL}$ scintillation vial was charged with $\mathbf{1}-\mathbf{V}_{6} \mathbf{O}_{7^{2-}}(0.050$ $\mathrm{g}, 0.0346 \mathrm{mmol})$ and acetonitrile $(4 \mathrm{~mL})$. A $2 \mathrm{~mL}$ acetonitrile solution of $\left[\mathrm{HNEt}_{3}\right] \mathrm{BF}_{4}(0.008 \mathrm{~g}$, $0.0423 \mathrm{mmol}, 1.22$ equiv) was added dropwise. The mixture was stirred for $15 \mathrm{~min}$, and then dried under reduced pressure. ESI-MS (-) $958\left(2-\mathbf{V}_{6} \mathbf{O}_{7^{1-}}\right), 960\left(3-\mathbf{V}_{6} \mathbf{O}_{6}{ }^{1-}+\mathrm{H}_{2} \mathrm{O}\right), 974 \mathrm{~m} / \mathbf{z}\left(3-\mathbf{V}_{6} \mathbf{O}_{6}{ }^{1-}+\right.$ $\mathrm{MeOH})$.

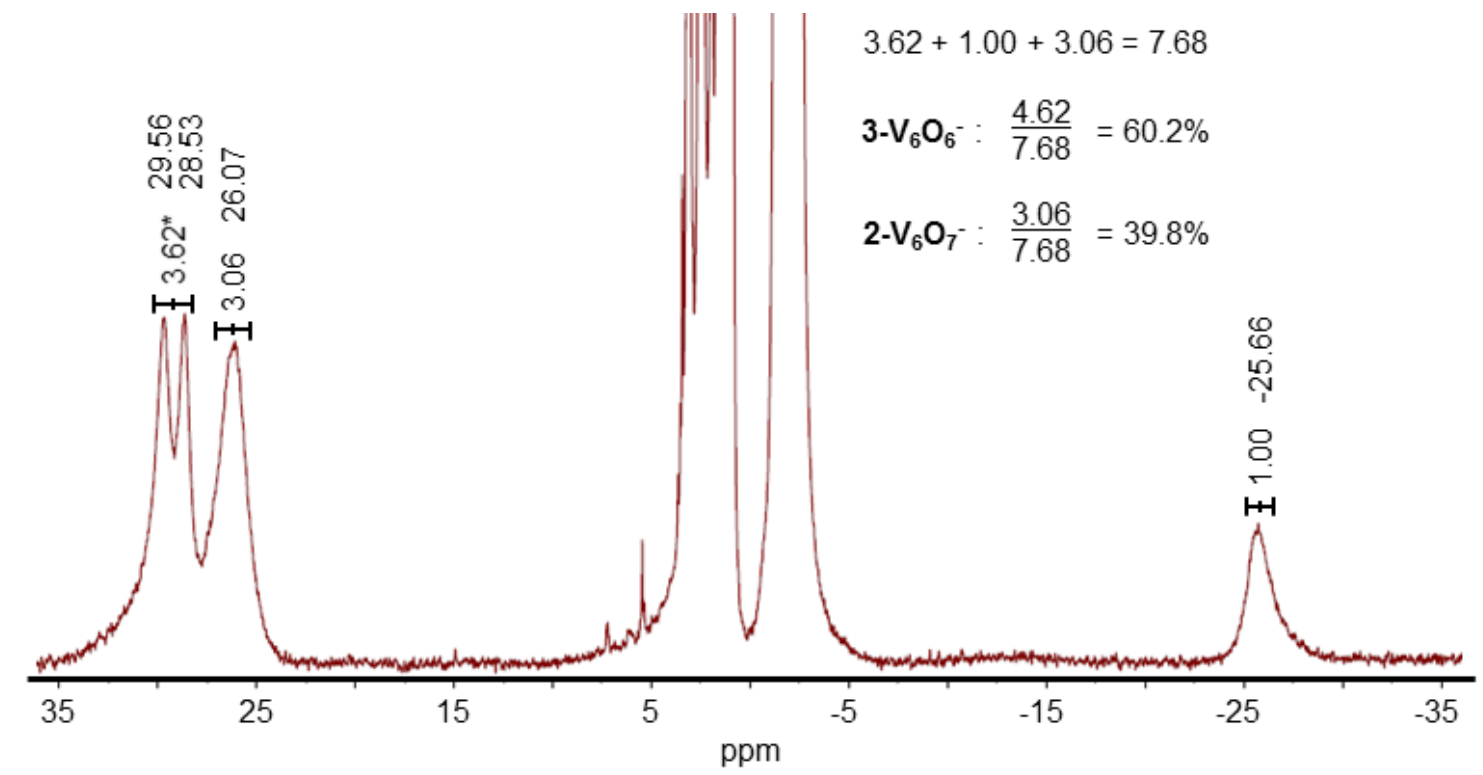

Figure S1. ${ }^{1} \mathrm{H}$ NMR spectrum of the addition of 1 equiv $\left[\mathrm{HNEt}_{3}\right]\left[\mathrm{BF}_{4}\right]$ to a solution of $1-\mathrm{V}_{6} \mathrm{O}_{7^{2-}}$ in $\mathrm{MeCN}-d_{3}$. Peaks are integrated at half-height to estimate relative amounts in solution. ${ }^{*} T$ The breadth of these paramagnetic signals, which correspond to $3-\mathbf{V}_{6} \mathbf{O}_{6}{ }^{1-}$, resulted in the individual resonances being integrated as one because they overlapped at both half heights. This introduces significant uncertainty with the deduced percentages of each species in the reaction mixture, as peak overlap results in increased integrated area for the individual shifts. 

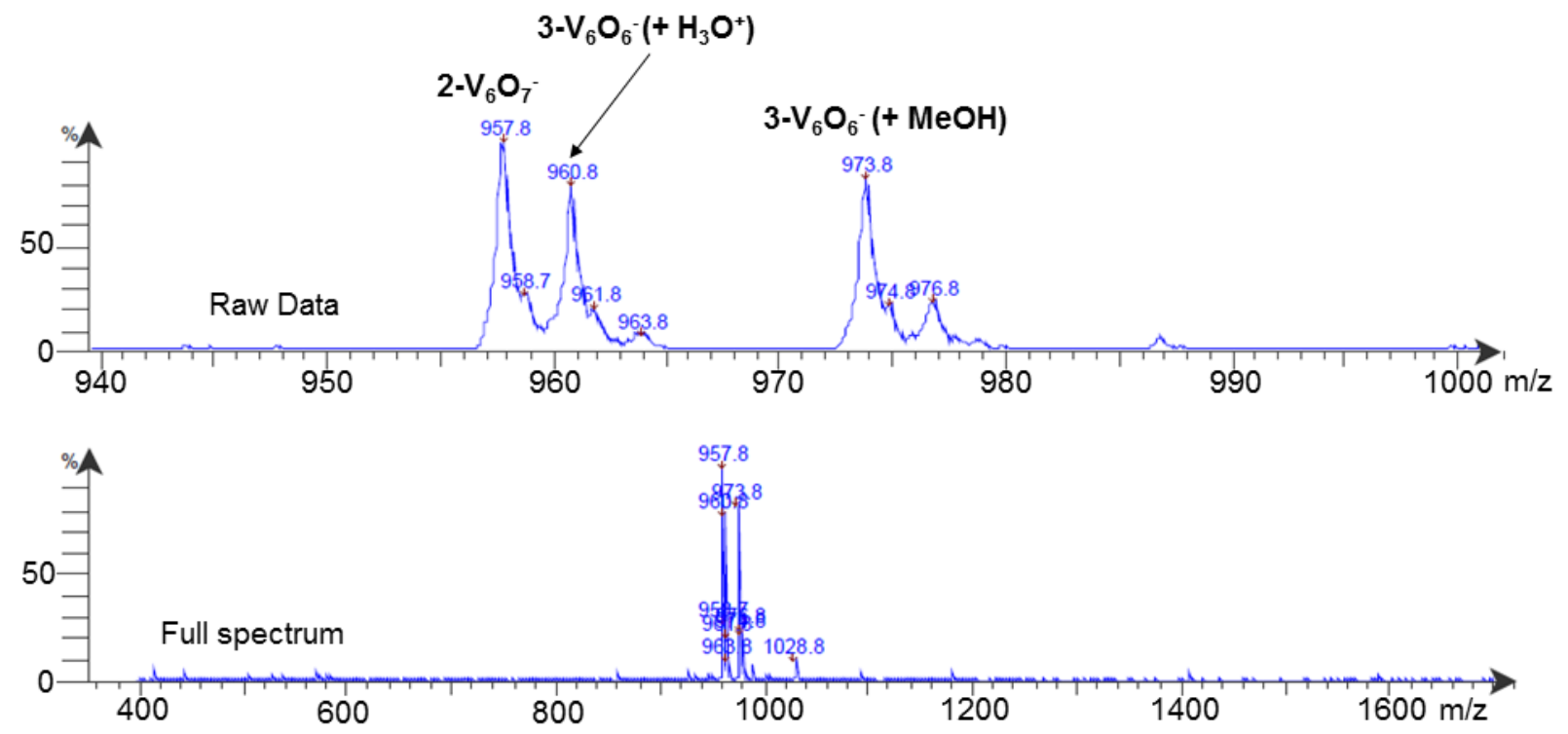

Figure S2. ESI-Mass Spectrum ((-)ve mode, low resolution) of reaction between $\mathbf{1 - V}_{6} \mathbf{O}_{7^{2-}}$ and $\left[\mathrm{HNEt}_{3}\right] \mathrm{BF}_{4}$.
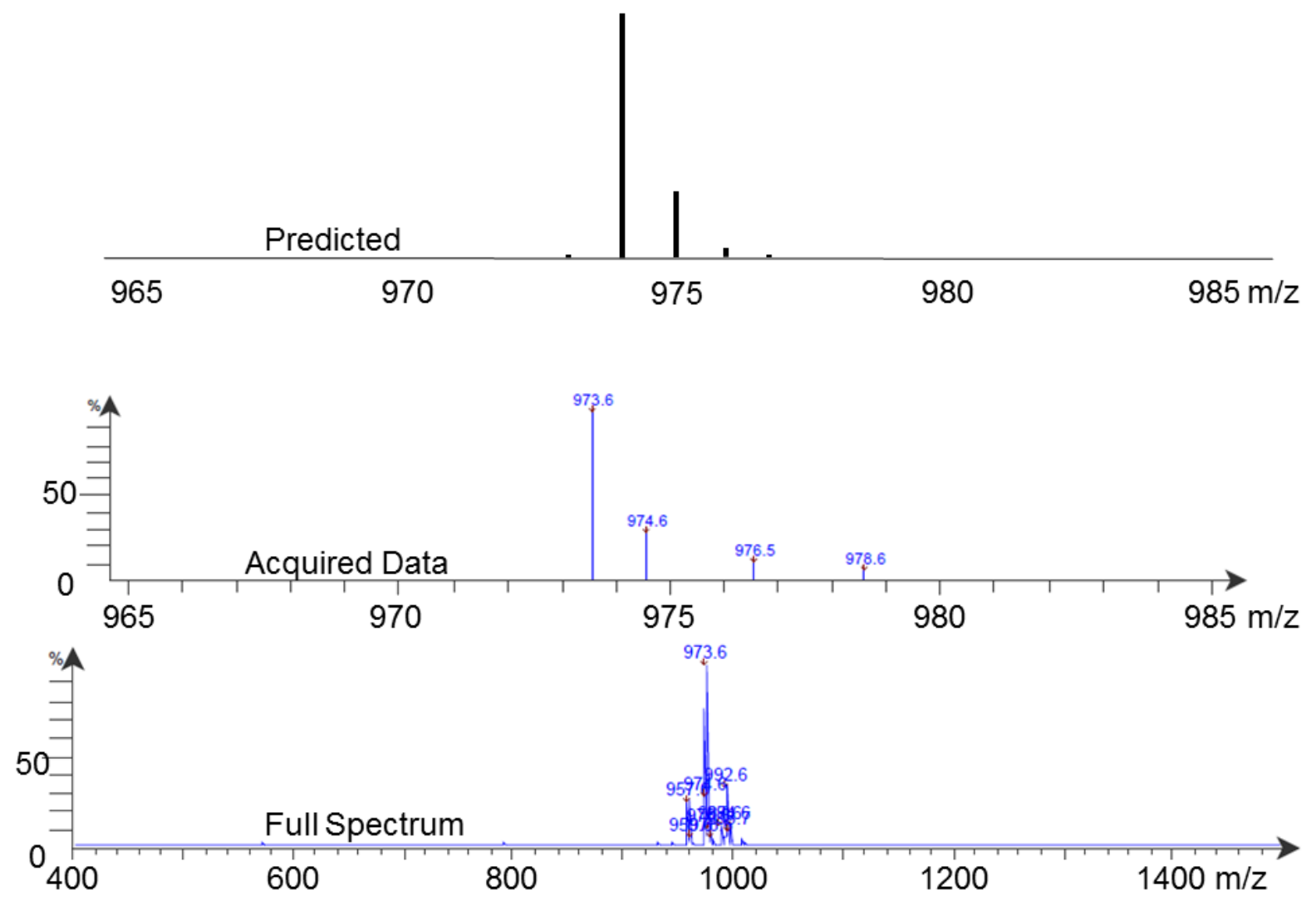

Figure S3. ESI-MS ((-)ve mode, low resolution) of $\mathbf{3}-\mathbf{V}_{6} \mathbf{O}_{6}{ }^{1-}$. 


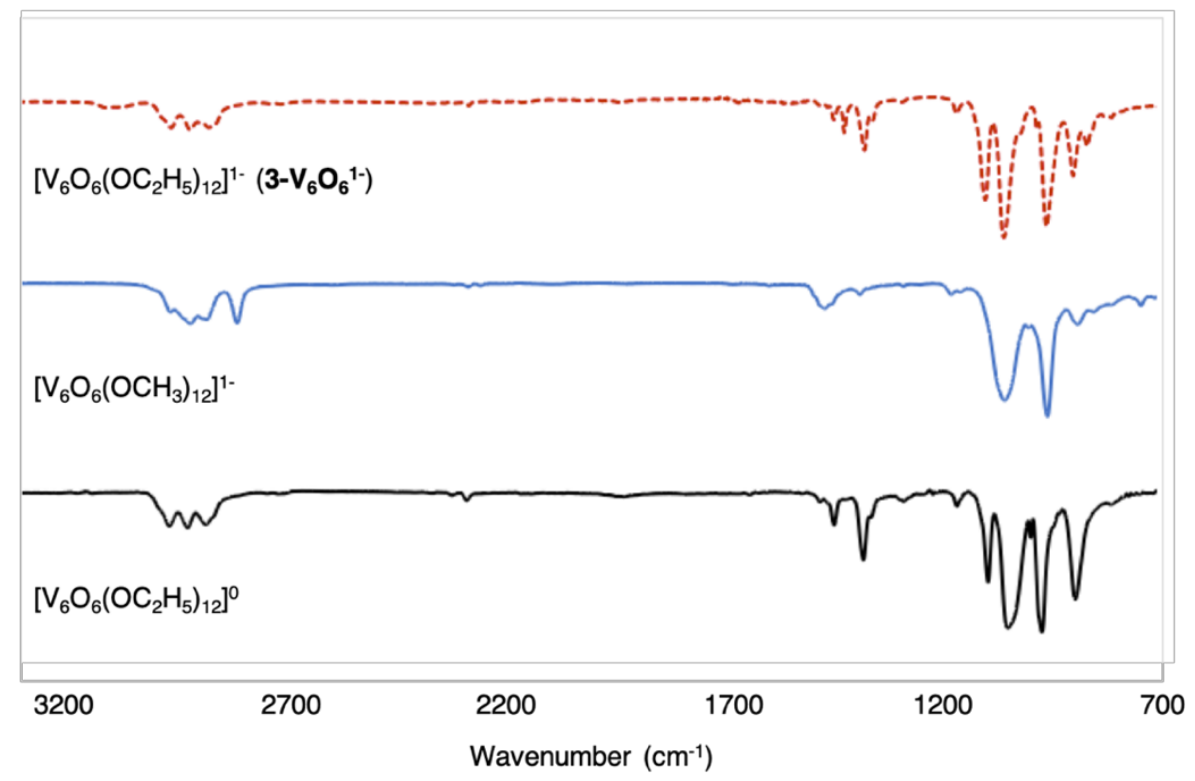

Figure S4. IR Spectra of $\mathrm{V}_{6} \mathrm{O}_{6}\left(\mathrm{OC}_{2} \mathrm{H}_{5}\right)_{12}{ }^{0}$ (bottom, black), $\left[\mathrm{V}_{6} \mathrm{O}_{6}\left(\mathrm{OCH}_{3}\right)_{12}\right]^{1-}$ (middle, blue), and 3$\mathbf{V}_{6} \mathbf{O}_{6}{ }^{1-}$ (top, red).

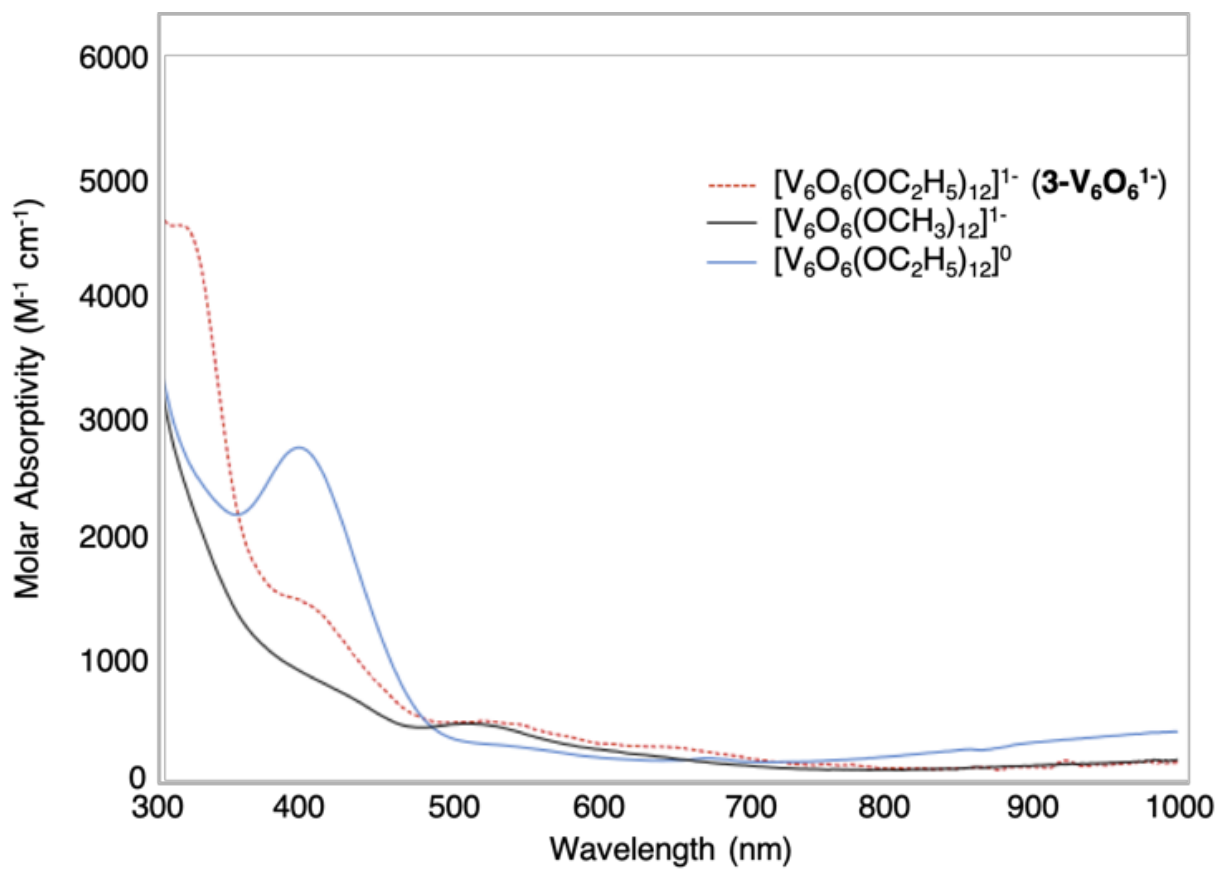

Figure S5. Electronic Absorption Spectrum of $\left[\mathrm{V}_{6} \mathrm{O}_{6}\left(\mathrm{OC}_{2} \mathrm{H}_{5}\right)_{12}\right]^{0}$ (black), $\left[\mathrm{V}_{6} \mathrm{O}_{6}\left(\mathrm{OCH}_{3}\right)_{12}\right]^{1-}$ (blue), and $3-\mathbf{V}_{6} \mathbf{O}_{6}{ }^{1-}$ (red) collected in dichloromethane at $21^{\circ} \mathrm{C}$. 
Table S1. Infrared and electronic absorption parameters for $\mathbf{3}-\mathbf{V}_{6} \mathbf{O}_{6}{ }^{1-},\left[\mathrm{V}_{6} \mathrm{O}_{6}\left(\mathrm{OCH}_{3}\right)_{12}\right]^{1-},{ }^{4}$ $\left[\mathrm{V}_{6} \mathrm{O}_{6}\left(\mathrm{OC}_{2} \mathrm{H}_{5}\right)_{12}\right]^{0},{ }^{5} \mathbf{1 -} \mathrm{V}_{6} \mathrm{O}_{7^{2-}},{ }^{6}$ and $\mathbf{2}-\mathrm{V}_{6} \mathrm{O}_{7^{1-}} .{ }^{6}$

\begin{tabular}{|c|c|c|c|c|c|c|}
\hline \multirow{3}{*}{$\begin{array}{l}\text { Compound } \\
3-\mathrm{V}_{6} \mathrm{O}_{6}{ }^{1-}\end{array}$} & \multirow{3}{*}{$\begin{array}{l}\text { Ox. State } \\
\text { Distrib. } \\
\text { VIIIVIV }_{5}\end{array}$} & \multicolumn{3}{|c|}{ Infrared Spectroscopy } & \multirow{2}{*}{\multicolumn{2}{|c|}{$\begin{array}{c}\text { Electronic Absorption Spectroscopy } \\
\text { Wavelengths and molar absorptivity of } \\
\text { IVCT Bands }\end{array}$}} \\
\hline & & $v\left(\mathrm{O}_{b}-\mathrm{R}\right)$ & $v\left(\mathrm{~V}=\mathrm{O}_{\mathrm{t}}\right)$ & $\Delta \mathrm{E}_{\mathrm{b}}$ & & \\
\hline & & $1049 \mathrm{~cm}^{-1}$ & $951 \mathrm{~cm}^{-1}$ & $98 \mathrm{~cm}^{-1}$ & -- & -- \\
\hline$\left[\mathrm{V}_{6} \mathrm{O}_{6}\left(\mathrm{OCH}_{3}\right)_{12}\right]^{1-}$ & 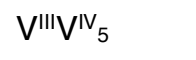 & $1047 \mathrm{~cm}^{-1}$ & $951 \mathrm{~cm}^{-1}$ & $96 \mathrm{~cm}^{-1}$ & -- & -- \\
\hline$\left[\mathrm{V}_{6} \mathrm{O}_{6}\left(\mathrm{OC}_{2} \mathrm{H}_{5}\right)_{12}\right]^{1-}$ & $\mathrm{V}^{\prime \prime \prime} \mathrm{V}^{\mathrm{IV}}{ }_{4} \mathrm{~V}^{\mathrm{V}}$ & $1041 \mathrm{~cm}^{-1}$ & $964 \mathrm{~cm}^{-1}$ & $77 \mathrm{~cm}^{-1}$ & $\begin{array}{l}394 \mathrm{~nm} \\
\left(2793 \mathrm{M}^{-1} \mathrm{~cm}^{-1}\right)\end{array}$ & $\begin{array}{l}1000 \mathrm{~nm} \\
\left(417 \mathrm{M}^{-1} \mathrm{~cm}^{-1}\right)\end{array}$ \\
\hline $1-\mathrm{V}_{6} \mathrm{O}_{7}^{2-}$ & $\mathrm{V}^{\mathrm{IV}_{6}}$ & $1055 \mathrm{~cm}^{-1}$ & $930 \mathrm{~cm}^{-1}$ & $125 \mathrm{~cm}^{-1}$ & -- & -- \\
\hline $2-\mathrm{V}_{6} \mathrm{O}_{7}{ }^{1-}$ & $\mathrm{VIV}_{5} \mathrm{~V}^{\mathrm{V}}$ & $1045 \mathrm{~cm}^{-1}$ & $945 \mathrm{~cm}^{-1}$ & $100 \mathrm{~cm}^{-1}$ & $\begin{array}{l}390 \mathrm{~nm} \\
\left(5750 \mathrm{M}^{-1} \mathrm{~cm}^{-1}\right)\end{array}$ & $\begin{array}{l}1000 \mathrm{~nm} \\
\left(1331 \mathrm{M}^{-1} \mathrm{~cm}^{-1}\right)\end{array}$ \\
\hline
\end{tabular}

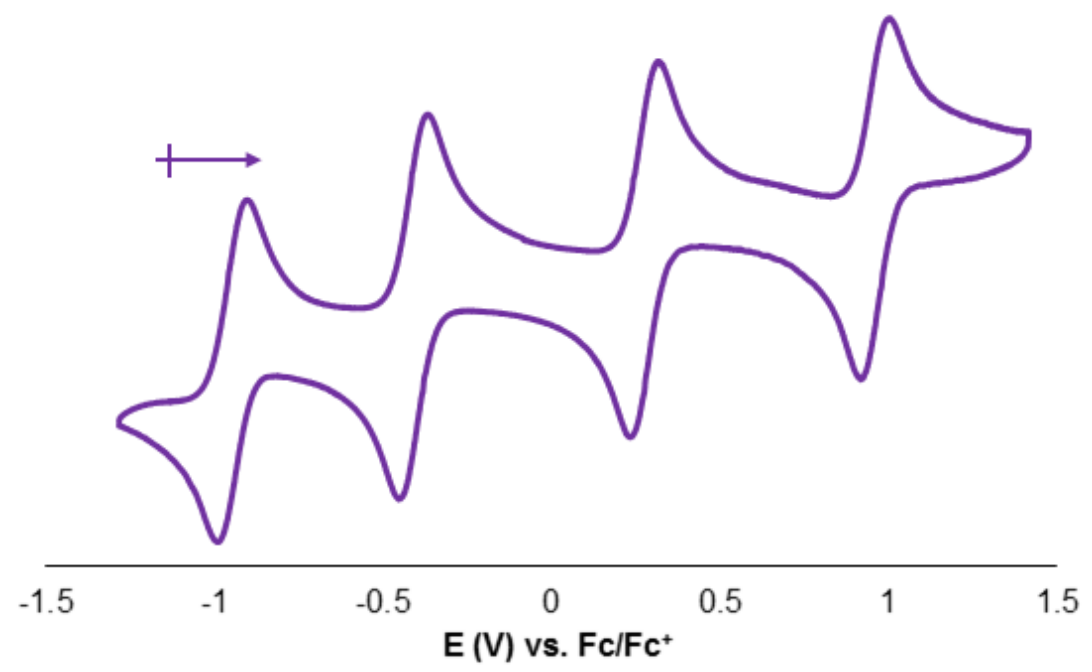

Figure S6. Cyclic Voltammogram of $1-\mathbf{V}_{6} \mathrm{O}_{7^{2-}}$ collected in acetonitrile with $0.1 \mathrm{M}\left[{ }^{\mathrm{n}} \mathrm{Bu}{ }_{4} \mathrm{~N}\right] \mathrm{PF} \mathrm{F}_{6}$ as the supporting electrolyte at a scan rate of $200 \mathrm{mVs}^{-1}$. 
Table S2. Redox Potentials of $1-\mathbf{V}_{6} \mathrm{O}_{7^{2-}}$ collected in acetonitrile with $0.1 \mathrm{M}\left[{ }^{n} \mathrm{Bu}_{4} \mathrm{~N}\right] \mathrm{PF}{ }_{6}$ as the supporting electrolyte.

\begin{tabular}{ccccc}
\hline \multicolumn{5}{c}{ Potentials of Redox Couples for 1- $\mathrm{V}_{6} \mathrm{O}_{7^{2-}}$} \\
Reference & {$\left[\mathrm{VIV}_{6}\right] \rightleftharpoons\left[\mathrm{VIV}_{5} \mathrm{VV}\right]$} & {$\left[\mathrm{VIV}_{5} \mathrm{VV}\right] \rightleftharpoons\left[\mathrm{VIV}_{4} \mathrm{VV}_{2}\right]$} & {$\left[\mathrm{VIV}_{4} \mathrm{VV}_{2}\right] \rightleftharpoons\left[\mathrm{VIV}_{3} \mathrm{VV}_{3}\right]$} & {$\left[\mathrm{VIV}_{3} \mathrm{VV}_{3}\right] \rightleftharpoons\left[\mathrm{VIV}_{2} \mathrm{VV}_{4}\right]$} \\
\hline $\mathrm{FC} / \mathrm{FC}^{+}$ & $-0.964 \mathrm{~V}$ & $-0.173 \mathrm{~V}$ & $0.258 \mathrm{~V}$ & $0.943 \mathrm{~V}$ \\
$\mathrm{SCE}^{a}$ & $-0.510 \mathrm{~V}$ & $0.281 \mathrm{~V}$ & $0.712 \mathrm{~V}$ & $1.397 \mathrm{~V}$ \\
$\mathrm{NHE}^{b}$ & $-0.268 \mathrm{~V}$ & $0.523 \mathrm{~V}$ & $0.954 \mathrm{~V}$ & $1.639 \mathrm{~V}$ \\
\hline
\end{tabular}

${ }^{a}$ Calculated from experimentally determined redox potentials versus $\mathrm{Fc} / \mathrm{Fc}^{+}$by considering this reference couple to be $0.454 \mathrm{~V}$ vs SCE. ${ }^{b}$ Calculated using the SCE potential vs. NHE: $0.242 \mathrm{~V}$.

Sample Calculation:

$$
\begin{aligned}
& -0.964 \mathrm{~V}\left(\mathrm{~V}_{\mathrm{Vl}}^{\mathrm{IV}} \rightleftharpoons \mathrm{V}_{\mathrm{v}}^{\mathrm{IV}} \mathrm{V}^{\mathrm{V}} \text { vs. } \mathrm{Fc} / \mathrm{Fc}^{+}\right) \\
& +0.454 \mathrm{~V}\left(\mathrm{Fc} / \mathrm{FC}^{+} \mathrm{vs} . \mathrm{SCE}\right){ }^{7} \\
& =-0.510 \mathrm{~V}\left(\mathrm{~V}_{\mathrm{VI}}^{\mathrm{IV}} \rightleftharpoons \mathrm{V}^{\mathrm{IV}}{ }_{\mathrm{v}} \mathrm{V}^{\mathrm{V}} \text { vs. SCE }\right) \\
& +0.242 \mathrm{~V} \text { (SCE vs. NHE) } 8 \\
& =-0.268 \mathrm{~V}\left(\mathrm{~V}_{\mathrm{VI}}^{\mathrm{V} \mathrm{V}} \rightleftharpoons \mathrm{V}^{\mathrm{IV}}{ }_{\mathrm{V}} \mathrm{V}^{\mathrm{V}} \text { vs. NHE }\right)
\end{aligned}
$$

References

(1) Spandl, J.; Daniel, C.; Brüdgam, I.; Hartl, H. Synthesis and Structural Characterization of Redox-Active Dodecamethoxoheptaoxohexavanadium Clusters. Angew. Chem. Int. Ed. 2003, 42, 1163-1166.

(2) Petel, B. E.; Matson, E. M. Conversion of NOx1- $(x=2,3)$ to NO Using an Oxygen-Deficient Polyoxovanadate-Alkoxide Cluster. Chem. Commun. 2020, 56, 555-558.

(3) McCarthy, B. D.; Dempsey, J. L. Decoding Proton-Coupled Electron Transfer with PotentialPKa Diagrams. Inorg. Chem. 2017, 56, 1225-1231.

(4) Petel, B. E.; Brennessel, W. W.; Matson, E. M. Oxygen-Atom Vacancy Formation at Polyoxovanadate Clusters: Homogeneous Models for Reducible Metal Oxides. J. Am. Chem. Soc. 2018, 140, 8424-8428.

(5) Petel, B. E.; Meyer, R. L.; Brennessel, W. W.; Matson, E. M. Oxygen Atom Transfer with Organofunctionalized Polyoxovanadium Clusters: O-Atom Vacancy Formation with Tertiary Phosphanes and Deoxygenation of Styrene Oxide. Chem. Sci. 2019, 10, 8035-8045.

(6) VanGelder, L. E.; Kosswattaarachchi, A. M.; Forrestel, P. L.; Cook, T. R.; Matson, E. M. Polyoxovanadate-Alkoxide Clusters as Multi-Electron Charge Carriers for Symmetric NonAqueous Redox Flow Batteries. Chem. Sci. 2018, 9, 1692-1699.

(7) Bao, D.; Millare, B.; Xia, W.; Steyer, B. G.; Gerasimenko, A. A.; Ferreira, A.; Contreras, A.; Vullev, V. I. Electrochemical Oxidation of Ferrocene: A Strong Dependence on the Concentration of the Supporting Electrolyte for Nonpolar Solvents. J. Phys. Chem. A 2009, 113, 1259-1267.

(8) Bard, A. J.; Faulkner, L. R. Electrochemical Methods: Fundamentals and Applications, 2nd Edition, 2nd ed.; Wiley Global Education, 2000. 